\title{
Analysing land cover and land use change in the Matobo National Park and surroundings in Zimbabwe
}

\author{
Valeska Scharsich ${ }^{\mathrm{a}, \mathrm{b}, *}$, Kupakwashe Mtata ${ }^{\mathrm{c}}$, Michael Hauhs ${ }^{\mathrm{a}, \mathrm{d}}$, Holger \\ Lange $^{\mathrm{d}, \mathrm{e}}$, Christina Bogner ${ }^{\mathrm{a}, \mathrm{d}}$ \\ ${ }^{a}$ Ecological Modelling, University of Bayreuth, DE \\ ${ }^{b}$ Soil Physics Group, University of Bayreuth, DE \\ ${ }^{c}$ Bayreuth International Graduate School of African Studies, University of Bayreuth, DE \\ ${ }^{d}$ BayCEER, University of Bayreuth, DE \\ ${ }^{e}$ Norwegian Institute of Bioeconomy Research, As, NO
}

\begin{abstract}
National parks are established to reduce human influence on nature and contribute to species conservation, biodiversity and ecological services. Other states of protection like the UNESCO world heritage sites, for example, are created for maintaining culturally important places or lifestyles. In the Matobo Hills (Zimbabwe) both states of protection are present, a national park and a world heritage site. In addition, the land outside the National Park belongs to two different systems of ownership, namely "common" (i.e. community-owned) and "not-common" (privately or governmentally owned) land.
\end{abstract}

In this paper, we investigated how the state of protection and the ownership affected the land use and land cover. We derived maps using Landsat images from 1989, 1998 and 2014 by supervised classification with Random Forests. To compensate for the lack of ground data we inferred past land

\footnotetext{
${ }^{*}$ Corresponding author. E-mail address: valeska.scharsich@uni-bayreuth.de
} 
use and land cover from recent observations combining photographs, Google Earth images and change detection. We could identify four classes, namely shrub land, forest, patchy vegetation and agricultural area.

The Matobo National Park showed a stable composition of land cover during the study period and the main changes were observable in the surroundings. Outside the national park, forest increased by about $7 \%$. The common lands have changed substantially and their agricultural area decreased. We attribute this development to the Fast Track Land Reform, which took place in the early 2000s. Our approach shows that combining information on recent land cover with change detection allows to study the temporal development of protected areas.

Keywords: land use and land cover, remote sensing, change detection, change vector analysis, Matobo National Park, Fast Track Reform of Zimbabwe

\section{Introduction}

National parks and protected areas in general play an important role in maintaining biodiversity, preserving endangered species and providing ecosystem services, such as water filtration or air cleaning (Gaston et al.,

5 2008; Belgacem et al., 2013). Today, protected areas cover over $15 \%$ of the global terrestrial and inland water areas and the goal for 2020 is to increase them to $17 \%$ (Juffe-Bignoli et al., 2014). Much effort is done to reach this goal. However, the official declaration of land as a protected area is often not sufficient and protection measures have to be implemented. In some cases the local inhabitants are resettled to establish a national park. As a 
consequence pastures might become inaccessible and game hunting is prohibited. This can decrease the acceptance of the park by local people and could result in disregarded boundaries by illegal hunting, illegal grazing of livestock or illegal farming, for example. Thus, protected areas are seldom isolated from their surroundings. Actually, changes in land use and land cover (LULC) around protected areas could influence their natural resources and ecological functioning (Jones et al., 2009). According to DeFries et al. (2007) an appropriate balance between conservation goals in protected areas and development of surrounding areas to improve human well-being is a core societal issue. Therefore, monitoring of LULC in protected areas need to include their surroundings.

To decide whether the state of protection or management of surrounding areas have a quantifiable effect on LULC, remote sensing provides a valuable data source. Particularly in order to observe large, heterogeneous or poorly accessible regions, remote sensing data are a good choice. Satellite images constitute the main tool to infer LULC all over the world and are used to detect changes and developments in various landscapes. Today, a broad range of satellite images and other remote sensing data are freely available and some data even date back to the middle of the last century. Therefore, it is possible to monitor and analyse the development of almost any region in the world and possibly assess anthropogenic impacts. In protected areas like national parks, the aim is to minimize these impacts.

In order to detect the effect of protection measures on LULC, two general approaches are possible. On the one hand, one can compare areas with 35 similar environmental conditions but with different states of protection. In 
most cases, this approach is based on a comparison of the protected area with its surroundings (Wasiolka and Blaum, 2011; Belgacem et al., 2013). On the other hand, it is possible to observe the temporal development of a protected area. For the latter purpose, time series of aerial photographs or of satellite images can be used for change detection, a wide field of research with many different methods (c.f. Tewkesbury et al. (2015); Lu et al. (2004) for an overview).

The most popular procedure for change detection is the post-classification comparison, in which separately classified satellite images are compared with regard to their respective LuLC classes (e.g. Bayarsaikhan et al., 2009). The main advantages of a post-classification comparison is its capability to provide complete information on LULC class changes and to reduce the influence of atmospheric and environmental differences between mutli-temporal images (Lu et al., 2004). The latter is particularly important when working with different satellite sensors like Landsat imagery, for example. However, the accuracy of a post-classification comparison depends on the quality of classification of every single image. One of the most widely used classifiers in remote sensing are Random Forests (Breiman, 2001). They show one of the best overall accuracies for a wide range of different data sets in comparison with over hundred other classifiers (Fernández-Delgado et al., 2014).

The main challenge for the classification is the availability of training data, in particularly for historical images. To deal with this issue we combine unsupervised clustering to identify spectrally similar pixels and change-vector analysis (Bovolo et al., 2012) to find pixels that remained unchanged over ${ }_{60}$ time. We use Google Earth images and recent ground photographs to identify 
LULC classes and train the classifier on unchanged pixels (Chen et al., 2012). To our knowledge, this approach to analyse LULC class changes in protected areas has rarely been used (Sieber et al., 2013; Gaveau et al., 2012), although it can be applied even when access to the region, for the reasons of local politics or difficult terrain, is not possible.

The aim of this study is to analyse changes of LuLC in the Matobo National Park and its surrounding area in Zimbabwe. The study area has a long history of conflicts regarding the land use and land ownership. Our focus is to investigate the effectiveness of the state of protection of the national park and the effect of land ownership in its surroundings.

\section{Material and Methods}

\subsection{Study area}

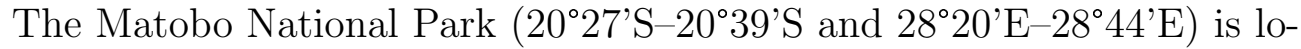
cated in the Matobo Hills in the southern part of Zimbabwe and covers an 75 area of $425 \mathrm{~km}^{2}$ (Ranger, 1989) (Figure 1). The Matobo Hills are characterised by a rolling countryside with granite inselbergs. The land cover ranges from fields and grazed areas, present mainly outside the national park, to shrub land and forest covering the park. The area surrounding the Matobo Hills is dominated by flat dry savannah and agricultural land.

Established in 1926, the Matobo National Park is the oldest national park of Zimbabwe with a long history of conflicts concerning the usage of the area. In its core area it remained continuously under protection. However, two parts in the south and in the east of the park were reattributed to common lands in 1953, a compromise for the local people. In 1982 the national park 
gained area in the north and remained unchanged since then.

A part of the Matobo Hills outside the national park is declared common land. This might have an effect on the land cover since the farming practice between these areas and the not-common land differs. The common land belongs to the community, in contrast to the privately or governmentally of the community. This field remains under control of the family for all generations until it is abandoned. This land is mostly cultivated by hand aided by livestock. In the literature the term common land is also referred to as communal land (Anderson et al., 1993).

95 in the North. In the Northwest, commercial farms run some camps for tourists. The forests outside the park are free to use for the villagers. Inside the park it is periodically allowed to harvest grass for one's own use or for selling. This strategy replaced in 1962 the periodical burning of the long grass in the national park. Outside the park fire is seldom used for bush control.

In the early 2000s, the Fast Track land reform changed the land ownership in large parts of Zimbabwe. As described by Alexander (2006) the reform started with the occupation of commercial farm land, which was mostly in possession of white farmers. The occupations were later legalized and at the end of 2001, 9 million hectares of land were acquired. In the following years 5 of the 9 million hectares, assigned for small-holder farming, were claimed mostly by households from communal areas. The remaining 4 million hectares were assigned for medium and large-scale commercial farming, yet in 
110 common lands east of $29^{\circ} 03^{\prime} 23^{\prime \prime}$ is missing, therefore, any results concerning the common lands refer to the land west of that longitude. 


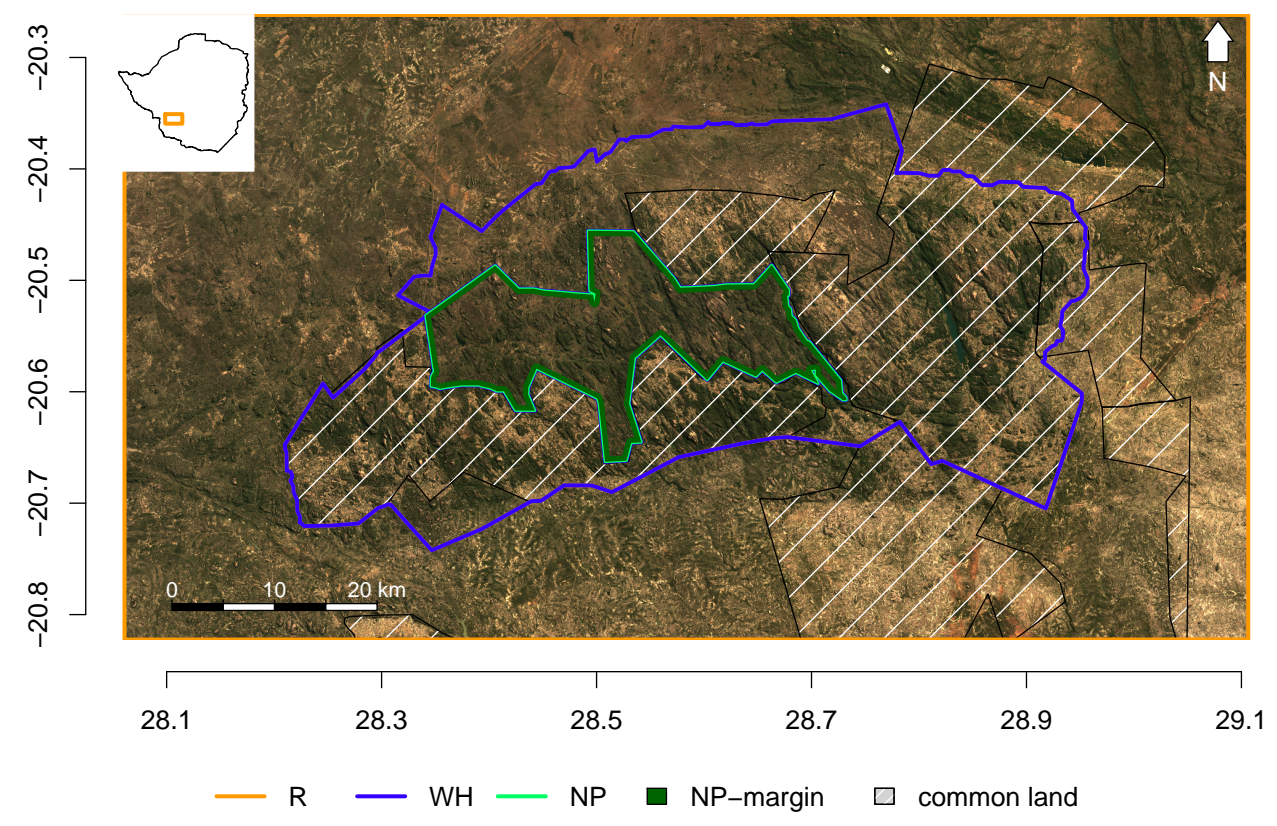

Figure 1: Partitioning of the study area with a Landsat image in the background, R: area outside the World Heritage Site, WH: World Heritage Site without national park, NP: Matobo National Park, NP-margin: the outer $500 \mathrm{~m}$ of the national park. The extension of the common lands east of $29^{\circ} 03^{\prime} 23^{\prime \prime}$ is unknown.

\subsection{Landsat images}

We analysed three Landsat surface reflectance images (downloaded from earthexplorer.usgs.gov on 11 March 2015) (spatial resolution $30 \mathrm{~m} \times$ $30 \mathrm{~m})$. They were acquired by L5 TM on 19th May 1989 and 10th May 1998 and by L8 OLI/TIRS on 15th May 2014. We used comparable bands in the images, namely $1-5$ and 7 in L5 TM and 2-7 in L8 OLI/TIRS. To guarantee good comparability, we selected the images in three steps. First, we chose images acquired between late April and early June. In this time the NDVI is declining, yet high, and live green vegetation is detectable (Supplementary 
Figure S1). In addition, the rainy season is already over and cloud contamination is minimal. Then, only images with less than $20 \%$ cloud cover were selected. Due to a scan line error, we avoided using Landsat 7 images acquired since 2003. In the third step, we only considered years where the amounts of precipitation in the preceding rainy seasons were comparable. Since no data for the study area itself where available, the duration of the rainy season and the total amount of precipitation were estimated from data (downloaded from www .ncdc . noaa.gov/cdo-web/datasets/GHCNDMS/ stations/GHCND : BC008948490/detail on 15 April 2015)measured in Francistown, Botswana, situated $125 \mathrm{~km}$ away from the Matobo National Park at a comparable altitude. This was the closest freely accessible meteorological station. The distance to the study area might result in some error concerning the exact amount of rainfall. Yet, we were interested in finding years with comparable rainy seasons, therefore only the relative amounts of precipitation were of interest.

\subsection{Preprocessing of surface reflectance data}

The Landsat surface reflectance images were already atmospherically corrected. Nevertheless, the visual impression of the image of 1998 differed from the other ones. This was due to larger reflectances in the blue band (Supplementary Figure S2). This difference will be considered in Section 2.4.2. However, it is not necessary to account for this in the post-classification comparison itself, since each image is classified separately.

165

Before further analysis, three additional preprocessing steps of the surface reflectance data were necessary. First, we masked water by using the cfmask band of Landsat image 1998. Masking the clouds was not necessary because 
the images were cloud free. Second, we checked that the image-to-image registration was satisfactory. Finally, we corrected the surface reflectance data topographically by the Minnaert method with slope correction (Goslee et al., 2011). We used the ASTER digital elevation model (ASTGTM2 S21E028 dem and ASTGTM2 S21E029 dem) with a spatial resolution of $29 \times 30.8 \mathrm{~m}$ that was resampled to match the resolution of the Landsat images.

\subsection{Determining the training data}

Acquiring reliable training data for classification is often a challenge ( $\mathrm{Lu}$ et al., 2004). To classify separately all three Landsat images with a supervised classification method we need a corresponding training data set for each image. In particular for the older images no recent field study could help to reconstruct these data reliably. In our work we follow the idea that training data inferred for the most recent satellite image can serve as a blueprint for the older ones. This means in particular that we transfer the LULC class of a pixel in the recent image to the corresponding pixel in the older images, provided that the pixel was identified as temporally unchanged. To find those pixels we combined two different methods, namely clustering and change vector analysis, as described below.

\subsubsection{Clustering of surface reflectance data}

For our study area, no reference data on the actual land use and land cover were available. Therefore we first clustered the 2014 Landsat image to find spectrally similar pixels and then used Google Earth and ground photographs from 2015 to identify the corresponding LULC classes in each cluster. The use of Google Earth to derive reference data has been suggested 
in many previous studies (e.g. Gong et al., 2013; Schneider et al., 2009; Knorn et al., 2009)

For clustering the data we used the method CLARA introduced by Kaufman and Rousseeuw (1990). It is similar to $k$-medoids clustering where data points are partitioned in $k$ clusters by minimizing the dissimilarities between all points in a cluster and a representative point that is chosen as cluster centre (i.e. the cluster medoid). In contrast to simple $k$-medoids, CLARA is suitable for large datasets (in our case 7421274 pixels). CLARA chooses randomly a subset of data points and calculates $k$ medoids on it. We set the subset size to 10000 pixels. Thereafter, each pixel of the entire dataset is assigned to the nearest medoid. The sampling of the subset is repeated 500 times to avoid a sampling bias. In every new repetition the sampled subset contains the medoids of the up to now best subset, i.e. the one with the minimal mean of dissimilarities of the sampled pixels to the closest medoid. Finally, the pixels are clustered using the medoids of the best subset and the resulting clustering is returned.

To obtain a suitable number of clusters $k$, we run CLARA for $k$ from 1 to 10 and selected an appropriate value visually with the so-called "elbow" method (Hastie et al., 2009). It calculates the within-cluster dissimilarities for every $k$, which naturally decreases with increasing $k$. In particular, the decrease for small $k$ is quite strong and flattens for large $k$. By identifying the position of the kink an appropriate $k$ can be inferred. In our case it led to $k=4$ or $k=5$. From visual inspection, $k=5$ resulted in highly fragmented clusters and some main landscape patterns were not adequately reproduced. Therefore, we chose $k=4$. 


\subsubsection{Change vector analysis - finding stable pixels}

To transfer the LuLC classes to pixels of older images we have to assure that the LULC class of the pixel remained unchanged during the study period. Therefore, we used change vector analysis to find unchanged pixels (Bovolo et al., 2012). For this method differences between a pair of Landsat images are calculated. It is essential that there is no general shift in the reflectance data between images because it would be interpreted as change. Due to the mentioned difference of the reflectance values (see Section 2.3) a

normalization was necessary and we subtracted the mean in every band as suggested by Bovolo et al. (2012). There might still be a slight spectral shift between the images, since they were acquired by sensors with different bandwidth, namely TM and OLI. However, we used the change vector analysis only to derive unchanged pixels without investigating the degree of change. ${ }_{230}$ Therefore, a possible slight shift is negligible.

With the normalized data, we calculated for both of the pairs 1989-2014 and 1998-2014 the length $\rho$ of the vector of differences between corresponding Landsat bands (also called spectral change vector) for each pixel

$$
\rho=\left(\sum_{b=1}^{B}\left(X_{b, 2}-X_{b, 1}\right)^{2}\right)^{1 / 2}
$$

where $X_{b, i}$ is the surface reflectance of band $b$ in image $i$ and $B$ is the number of bands compared in the two Landsat images. This measures the degree of change, i.e. the larger $\rho$, the larger the change. Since the distribution of $\rho$ is unknown, we fit the sum of two Gaussian distributions to its histogram (Figure 2) to infer a threshold for both image pairs (Bovolo et al., 2012). A pixel belonging with probability of at least 0.6 to the Gaussian with the 

(2012), where 0.5 instead of 0.6 was used. Since these pixel should serve as training data, a higher probability for being really unchanged is desirable. With these settings we derived the threshold $\rho=0.045$ for both pairs 19892014 and 1998-2014.
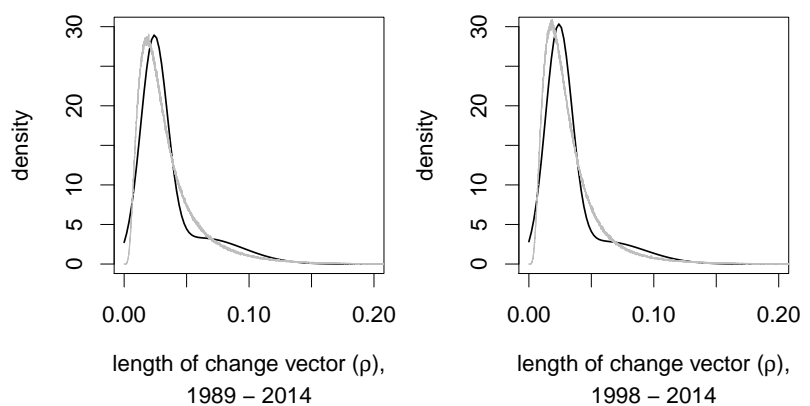

Figure 2: Distribution of $\rho$ (gray line) and fit consisting of two Gaussians (black line). $\rho$ is the length of the change vector, which is calculated separately for each pixel from the difference between the surface reflectance in each band of two Landsat images. It describes the degree of change in a pixel.

All pixels classified as unchanged could serve as training pixels with the corresponding LULC class of the image from 2014. However, to avoid isolated pixels and control the classification of the pixels the unchanged pixels were plotted and examined visually. The potential training pixels were compared with an actual Google image to get an impression of the LULC of the different classes identified by CLARA. We chose pixels for training data, if they belonged to homogeneous regions (i.e. the adjacent pixels were of the same class) and the LULC class of the region corresponded to the main LULC of the Google image. The location of these training pixels and their LuLC class was transferred to the images of 1989 and 1998. For each of the three years the 


\subsection{Classification of land use and land cover}

We used Random Forests (Breiman, 2001) as supervised classification algorithm to determine the LULC classes for all pixels in the Landsat images. The number of derived training pixels varied for each LULC class and was at least 3503. To avoid an over- or under-representation of certain LULC classes in the training data we chose randomly the maximal possible number of 3503 pixels per LULC class for each Landsat image.

The Random Forests classifier has three hyperparameters, namely the number of variables sampled randomly at each tree node from the set of possible variables (mtry), the minimal size of the terminal nodes (nodesize) and the number of trees in the forest (ntree). The variability in model performance for mtry between 1 and 5 spectral bands (i.e. maximum number of spectral bands minus one) was minor and we set this hyperparameter to its default value $m t r y=2$ (i.e. the square root of possible variables) as recommended by Breiman (2003). For the other two hyperparameters an overfitting is hardly possible and the variability of the performance is known to be low provided the number of trees is sufficiently large (Breiman, 2001; Strobl et al., 2009). Therefore, we used ntree $=1000$ (Kuhn and Johnson, 2013) and the default nodesize $=1$ (Breiman, 2001).

The derived classified images were used in a post-classification comparison. This method allows for an intuitive interpretation of the results due to pixel-to-pixel comparisons and provides a complete change matrix to identify 

consistency was calculated as follows:

$$
m C=\frac{\sum_{j=1}^{4} n_{j j}}{\sum_{k, j=1}^{4} n_{k j}}
$$

where $\left(n_{k j}\right)$ is the $(4 \times 4)$-confusion matrix. This matrix shows the number of pixels in predicted classes (rows) versus given classes (columns). Accordingly, the diagonal elements display the correctly classified pixels and the rest describes which classes were confused in the prediction.

Additionally, we calculated the per-class effectiveness (e.g. Sokolova and Lapalme, 2009) defined as 


$$
p E_{i}=\frac{n_{i i}+\sum_{\substack{k, j=1 \\ k, j \neq i}}^{4} n_{k j}}{\sum_{k, j=1}^{4} n_{k j}}
$$

where $p E_{i}$ is the per-class effectiveness for one of the four LULC classes $i$ ( $i=$ $1, \ldots, 4)$, and $\left(n_{k j}\right)$ the confusion matrix as in (2). The per-class effectiveness allows to analyse the consistency of the classification model for each LULC class separately.

We avoided to calculate the common index of agreement $\kappa$, which according to Pontius and Millones (2011) "gives information that is redundant or misleading". Instead, as suggested by these authors, we calculated for the model performance the two error measures "allocation disagreement"

$$
A=\sum_{g=1}^{J} \min \left[\left(\sum_{i=1}^{J} p_{i g}\right)-p_{g g},\left(\sum_{j=1}^{J} p_{g j}\right)-p_{g g}\right]
$$

and "quantity disagreement"

$$
Q=\frac{1}{2} \sum_{g=1}^{J}\left|\left(\sum_{i=1}^{J} p_{i g}\right)-\left(\sum_{j=1}^{J} p_{g j}\right)\right|
$$

where $\left(p_{i j}\right)$ is the estimated population matrix. Because we sampled each class equally, it can be calculated as

$$
p_{i j}=\frac{n_{i j}}{J \sum_{j=1}^{J} n_{i j}}
$$

where $J$ is the number of determined clusters $\left(J=4\right.$ in our case) and $\left(n_{k j}\right)$ the confusion matrix as in (2). 
The quantity disagreement corresponds to the error caused by an underor overestimation of classes, whereas the allocation disagreement describes the error caused by a suboptimal assignment of the classes to the pixels. Allocation disagreement and quantity disagreement sum up to the consistency

315 error of the classification (Pontius and Millones, 2011). Therefore, they are suitable to explain the consistency error of the classification in a more detailed way than the index of agreement $\kappa$.

Each of these errors was calculated for each of the repetitions of the 5-fold cross-validation, so that their variability could be assessed.

All calculations were done in R ( R Core Team, 2016). For georeferencing and topographical correction we used the add-on package landsat (Goslee et al., 2011); for resampling the add-on package raster (Hijmans, 2016) and for classification the add-on packages randomForest (Liaw and Wiener, 2002) and caret (Kuhn, 2016).

\section{Results and Discussion}

\subsection{Land use and land cover classes}

We determined four LuLC classes in the study area (Figure 3). Their names represent our visual impression of the main land cover and do not refer to internationally agreed definitions. Because we lack field reference data, more detailed information about the LULC classes (i.e. tree height or density) is unavailable. Since in nature most LULC classes merge into one another, the derived LULC classes also contain mixed pixels.

shrub land: This LULC class is characterised by grass with some shrubs and/or isolated trees. 
patchy vegetation: A pixel belonging to this class consists in most cases of more than one land cover, e.g., the edge of agricultural areas covered with isolated trees or shrubs and streets lined with trees or shrubs. Furthermore, grey rocks and shrub land with very sparsely grass, also agricultural area: This class describes mainly areas influenced by humans. These are primarily agricultural fields, but also wide streets, settlements and heavily grazed meadows. In addition, bright bare soils and shiny hilltops also fall into this class.

\subsection{Model consistency}

The model consistencies for all three Landsat images were very high and their standard deviations small (Table 1). Additionally, the training data of all four LULC classes were equally well predicted and the per-class effectiveness always exceeded $94 \%$. This is in favour of the model, yet it does not describe the overall accuracy, obtained by comparing the predicted LULC classes with ground data.

We cannot quantify the overall accuracy due to the lack of sufficient ground data for the study area. However, an evidence for a good classification might by the core area of the national park. It exists since 1926, therefore we expected only slight changes in the composition of the LULC classes in the studied period. Indeed, the classification results show quite similar composition for all three years (c.f. Section 3.4). 

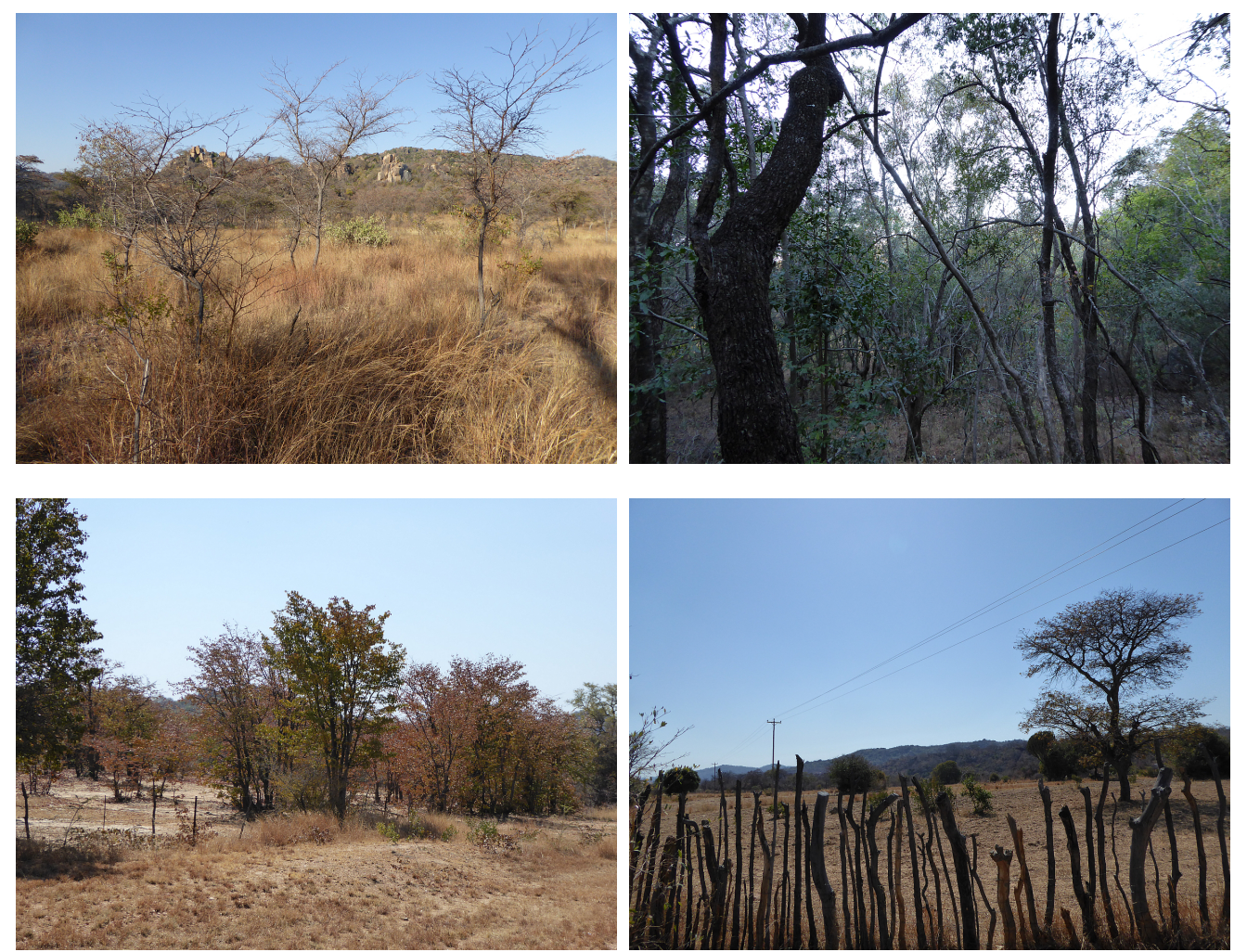

Figure 3: Photographs illustrating the four land use and land cover classes: shrub land (top left), forest (top right), patchy vegetation (bottom left) and agricultural area (bottom right). (CKupakwashe Mtata) 
The other parameters calculated to infer the model consistency also indicated good results. The allocation disagreement was larger than the quantity

360

Table 1: Mean model consistency from a 5-fold cross-validation (20 repetitions) with standard deviations in parenthesis. $p E_{i}$ : per class effectiveness for shrub land $(i=1)$, forest $(i=2)$, patchy vegetation $(i=3)$ and agricultural area $(i=4), m C$ : model consistency, $Q$ : quantity disagreement and $A$ : allocation disagreement. All numbers are in percent.

\begin{tabular}{lrrr}
\hline & 1989 & 1998 & 2014 \\
\hline$m C$ & $94.11(0.07)$ & $93.70(0.10)$ & $99.60(0.02)$ \\
$p E_{1}$ & $94.94(0.08)$ & $94.73(0.08)$ & $99.69(0.02)$ \\
$p E_{2}$ & $98.74(0.04)$ & $98.72(0.04)$ & $99.94(0.01)$ \\
$p E_{3}$ & $95.37(0.06)$ & $94.98(0.09)$ & $99.65(0.02)$ \\
$p E_{4}$ & $99.17(0.03)$ & $98.97(0.03)$ & $99.91(0.01)$ \\
$Q$ & $0.50(0.03)$ & $0.57(0.05)$ & $0.10(0.02)$ \\
$A$ & $5.35(0.08)$ & $5.68(0.11)$ & $0.30(0.02)$ \\
\hline
\end{tabular}

\subsection{General changes in land use and land cover}

The results of the classification are shown in Figure 4. For the whole study area we observed a reforestation. During the study period forest increased 
by about $7 \%$, whereas patchy vegetation decreased by about $6 \%$ (Table 2 ). 370 The proportions of the other two LULC classes shrub land and agricultural area remained quite stable. Overall, the changes in 1989-1998 and in 19982014 and their magnitude were comparable. Nevertheless, the changes in the two different time spans should not be compared in detail, since they are of quite different time lengths, 9 and 16 years respectively. We refrain from calculating an annual rate because it would imply a constant change rate that we could not support with data.

Table 2: Composition of LULC classes in percent in the whole study area.

\begin{tabular}{lccc}
\hline LULC & 1989 & 1998 & 2014 \\
\hline shrub land & 36.42 & 37.34 & 37.10 \\
forest & 21.32 & 22.83 & 27.83 \\
patchy vegetation & 28.55 & 26.26 & 23.01 \\
agriculural area & 13.71 & 13.57 & 12.06 \\
\hline
\end{tabular}

Comparing the images of 1989 and 2014 pixel-wise, over $38 \%$ of all pixels in the study area have changed their LulC classes (Table 3). However, this strong change could not be observed in the temporal comparison of the total LULC class proportions (Table 2), since many pixels leaving one class were replaced by other pixels. One example is the LuLC class patchy vegetation. Only $13 \%$ of the class remained stable and $11 \%$ changed to shrub land. At the same time $5 \%$ of pixels changed in the reverse direction. This fluctuation is partly caused by pixels belonging to different LULC classes, yet showing quite similar spectral characteristics. A slight change in vegetation cover could lead 
(a) 1989

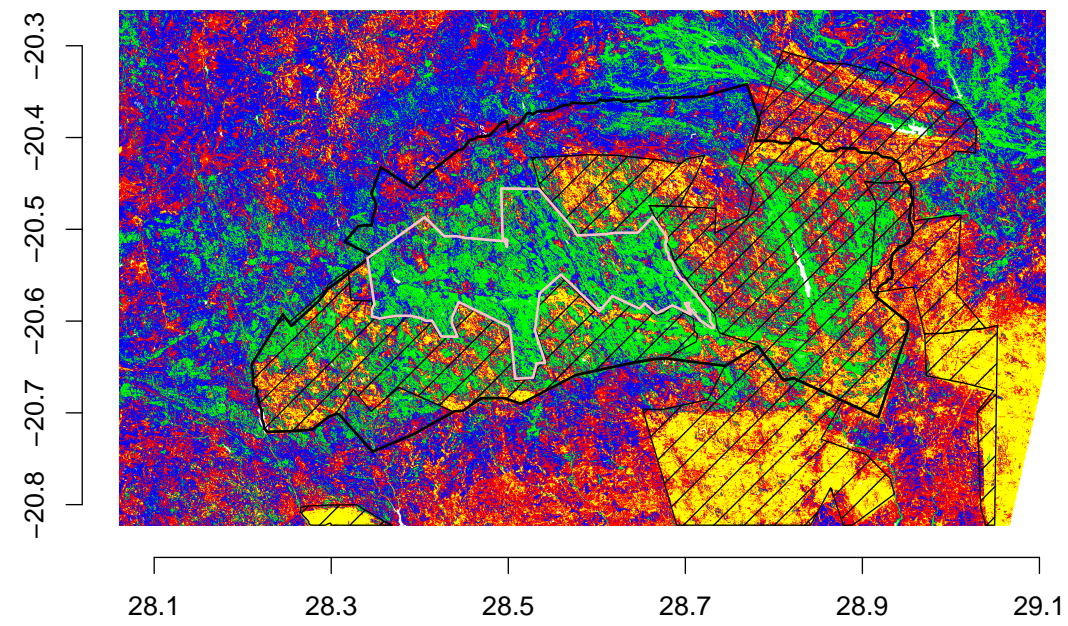

(b) 1998

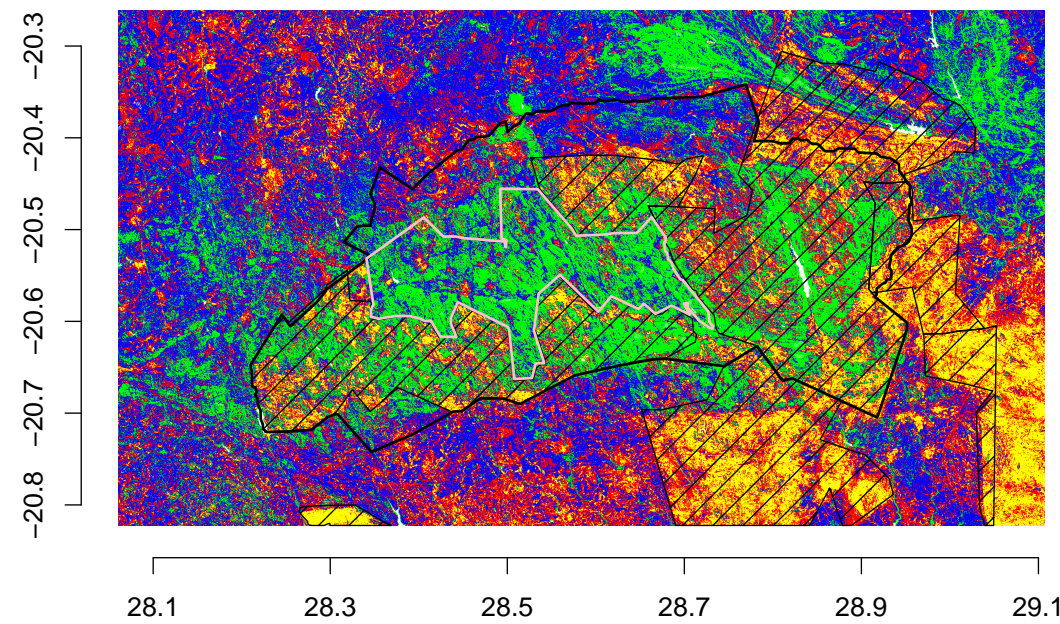

(c) 2014

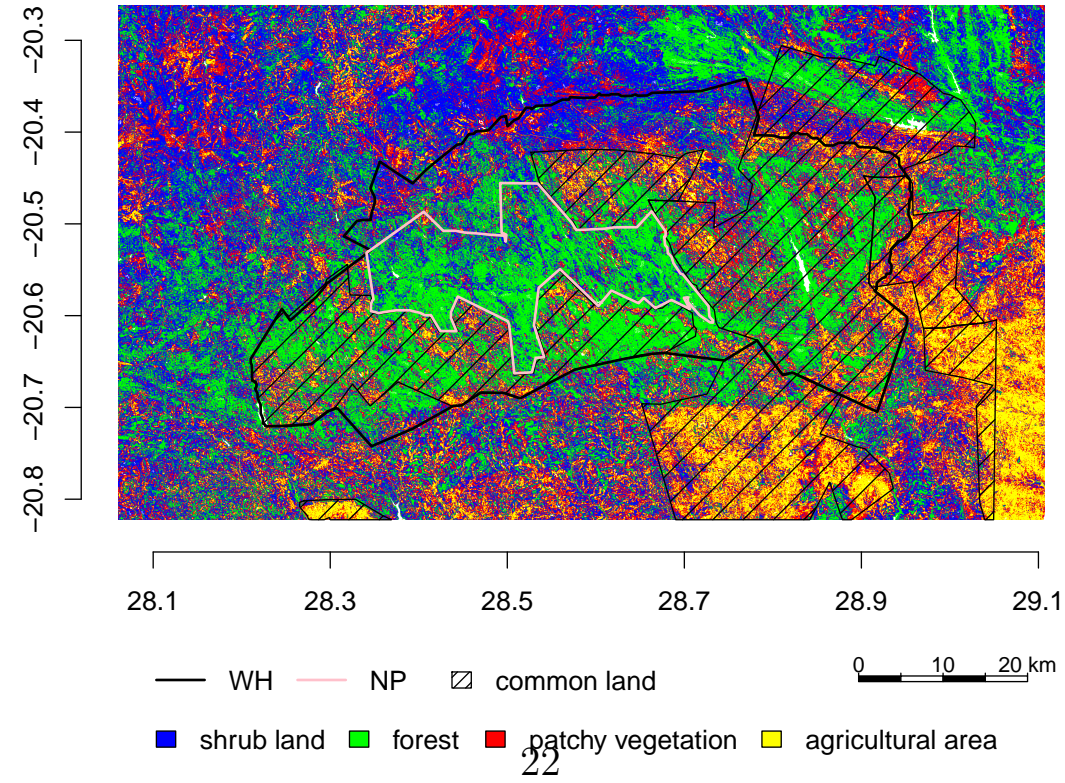

Figure 4: Spatial distribution of four LULC classes in the study area. The corresponding LULC classes are: 1: shrub land, 2: forest, 3: patchy vegetation, and 4: agricultural area. 
to a change in LuLC classes. One part of the $38 \%$ of change could probably be attributed to the overall classification error that we cannot determine as described in section 3.2. Since we are comparing two separately classified images, the error of the comparison equals approximately the product of the two respective classification errors. This is one of the drawbacks of the post-classification method (Coppin et al. 2004).

Table 3: Pixel-wise changes of the LuLC classes shrub land, forest, patchy vegetation and agriculural area in percent for the whole study area.

\begin{tabular}{lrrrr}
\hline LULC & shrub land & forest & patchy veg. & agric. area \\
1989 & 2014 & 2014 & 2014 & 2014 \\
\hline shrub land & 22.06 & 8.92 & 4.96 & 0.49 \\
forest & 3.42 & 17.60 & 0.25 & 0.05 \\
patchy vegetation & 10.93 & 1.42 & 13.32 & 2.88 \\
agricultural area & 0.90 & 0.05 & 4.47 & 8.30 \\
\hline
\end{tabular}

\subsection{Changes depending on state of protection}

The three subregions R (residual area), WH (World Heritage Site without National Park) and NP (National Park) represent three different states of protection. The composition of LULC classes in NP differed substantially from its surroundings (Figure 4 and Figure 5). Actually, inside the park forest dominated and the LULC class agricultural land barely existed. In contrast, outside NP the distribution of the LULC classes was more balanced. The proportions of LULC classes in the National Park remained rather stable, only a small amount of shrub land evolved to forest. This might be caused by 
the fact that the main part of the national park with the actual boundaries is under protection since 1926. The differences between WH and NP, although belonging to the same landscape, namely the Matobo Hills, do not surprise. Indeed while $\mathrm{WH}$ is used for agriculture, it is prohibited in NP.

The composition of WH and R differed, however, the temporal changes were comparable except for agricultural area (Figure 5). Both regions showed an increasing proportion of forest (5\% in WH and $7 \%$ in R) and a decreasing proportion of patchy vegetation ( $6 \%$ in both regions). These changes were not induced by a direct reforestation of patchy vegetation, but mainly by a transition from patchy vegetation to shrub land and from shrub land to forest (see Section 2.2 in the online Supplementary Material). The agricultural area decreased in WH considerably more than in R. The main changes occurred between 1998 and 2014. We do not attribute this change to the establishment of the World Heritage Site, since the decrease of agricultural area only occurred in the common lands, which represent the main part of WH. This will be discussed in section 3.6.

Since the development in $\mathrm{WH}$ and $\mathrm{R}$ was comparable and the differences in composition were already present in 1989, they were probably caused by differences in landscape. The declaration of the World Heritage Site in 2003, might have an effect on the composition of future LulC classes. Yet, the temporal development in WH until 2014 followed the overall trend in the study area. A more detailed analysis needs longer observations to detect whether the state of World Heritage Site has any effect on the LULC classes in the Matobo Hills. 


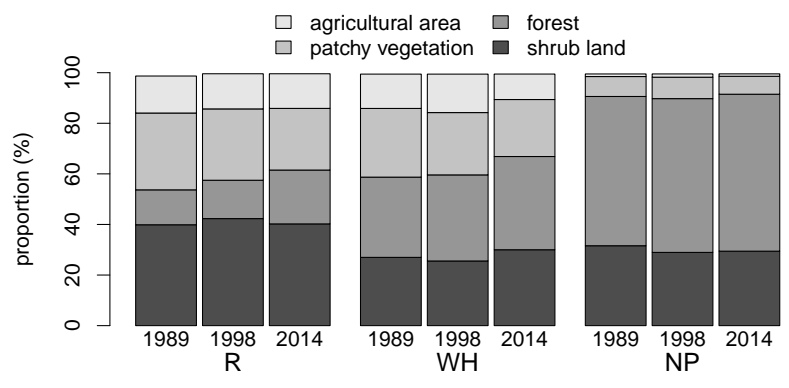

Figure 5: Fractions of the four LuLC classes in the years 1989, 1998 and 2014 for the regions: Area outside the World Heritage Site (R), World Heritage Site without national park (WH), national park (NP). By adding the pixels with missing values (see south east of the image of 1989, Figure 4a) every bar sums up to 1 . The exact numbers can be found in Section 2.2 in the online Supplementary Material.

\subsection{Changes along the national park boundary}

We divided the national park into two zones, a $500 \mathrm{~m}$ margin and a core area to assess a possible effect of fence damaging and grazing in the park. The main difference between the margin and the core was the proportion of shrub land and forest (Figure 6). The margin contained approximately $2 \%$ less shrub land than the core, yet 3\% more forest. Although these differences remained stable from 1989 to 2014, the absolute fractions of the LULC classes changed through time. While shrub land decreased in 1998, forest and patchy vegetation increased.

The margin is known to be occasionally grazed, therefore we expected a decrease of the LuLC classes shrub land and forest. However, only shrub land showed a lower proportion in the margin than in the core, whereas the area of forest was even larger. Therefore, the difference might be due to land characteristics and we cannot attribute it with certainty to illegal grazing in 


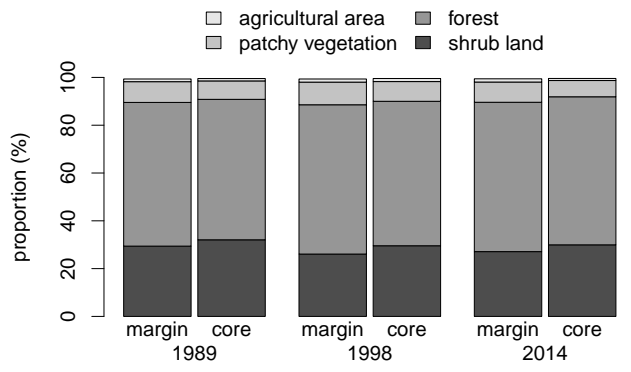

Figure 6: Fractions of LULC classes for a $500 \mathrm{~m}$ margin of NP and the core area. The exact numbers can be found in Section 2.2 in the online Supplementary Material.

the park.

\subsection{Spatial differences depending on ownership}

The spatial differences with regard to ownership could be best seen from the sharp boundaries of the common land in the south east of the study area, in particular in 1989 (Figure 1 and Figure 4). This implies distinct composition of LULC classes in common land versus not-common land, especially in

the residual area not containing WH (Figure 7). The not-common land was dominated by shrub land, whereas the fraction of agricultural land was quite small. Within the common lands the main difference of $\mathrm{R}$ and $\mathrm{WH}$ was the fraction of forest, which is quite prominent in WH. In contrast, common land in $\mathrm{R}$ was dominated by agricultural area and only a small area was covered by forest.

The difference between common and not-common land might be due to different land management. The common land is characterised by smallholder farming, where the average size of land for one household is about five hectares. On the contrary, the not-common land is divided into fields of 169 hectares, on the average (Sudhir Wanmali, 1997). The fragmentation of the 
common land might be a reason for its frequent assignment to agricultural area, in contrast to the not-common lands, which is mostly assigned to patchy vegetation.

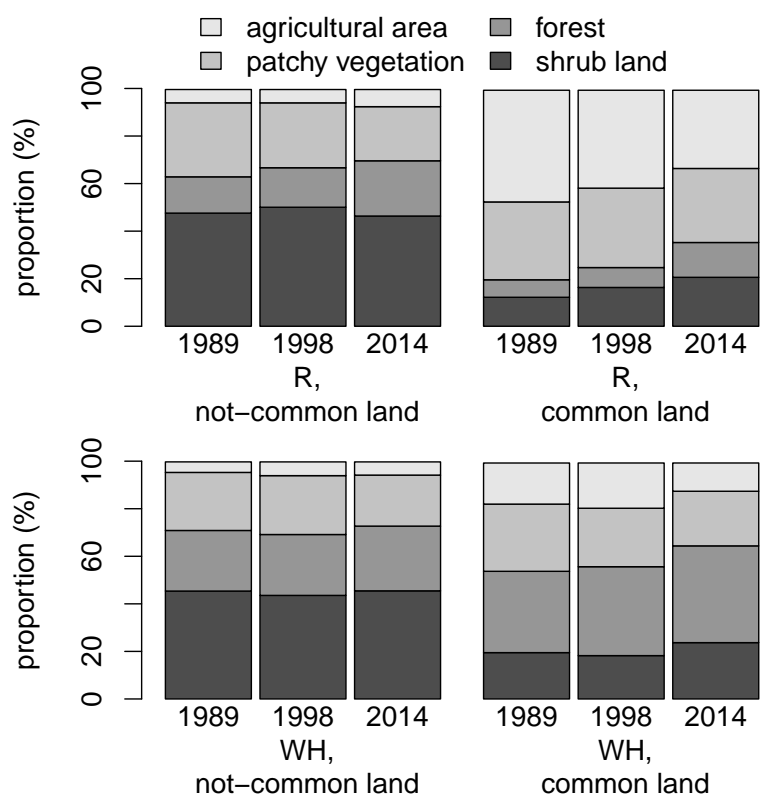

Figure 7: Fractions of the four LULC classes in the years 1989, 1998 and 2014 for the regions: Area outside the World Heritage Site (R), and World Heritage Site without national park $(\mathrm{WH})$, both distinguished for common land and not-common land. Region $\mathrm{R}$ was reduced to the part of $\mathrm{R}$ west of $29^{\circ} 03^{\prime} 23^{\prime \prime}$ due to missing information on the extension of common land in the East. The exact numbers can be found in Section 2.2 in the online Supplementary Material.

\subsection{Temporal differences depending on ownership} lands in R and WH and the not-common lands in R (Figure 7), which corresponds to the change observed for the whole study area. The main changes 
concerned the common lands. Indeed, the boundaries of the common lands became more and more fuzzy and the amount of agricultural area decreased

1998. This could be the consequences of the New National Policy from 1990. Following Alexander (2006) it announced to declare about 5 million hectares 
of former commercial farms for resettlement, yet the announcements were realized very slowly and incompletely. In contrast, the decrease of agricultural area was not observable in the common lands in WH. Therefore the political activities could only partly explain this development and an additional local cause seems probable.

\section{Summary and Conclusions}

In this study we analysed spatial and temporal effects of the state of protection and ownership on the LULC in the Matobo National Park and its surroundings in Zimbabwe. We used remote sensing data and combined several methods to overcome missing field data.

We found large differences in the composition of LuLC classes between the national park and its surroundings. The differences between the World Heritage Site, excluding the National Park, and the National Park itself are likely due to the state of protection of the national park, which exists since 1926. Indeed, both areas belong to the Matobo Hills. In contrast, the differences between the World Heritage Site, excluding the national park, and the surrounding area were smaller and occurred probably due to differences in landscape.

The temporal development in the study area, excluding the national park, is characterised by an increase of forest and an important decrease of agricultural area in the common lands. We attribute this development to the Fast Track Reform, which started in 2000. The composition of LULC classes in the national park remained quite stable. This is in accordance with the long history of the park. The differences between the margin and core area 
of the park remained comparable and are relatively small. Therefore we do not expect them to be caused by fence damaging and illegal grazing. They probably represent differences in the landscape.

In our analysis, we used relatively broad LULC classes, especially the LULC classes patchy vegetation and agricultural area included pixels of various types. To improve the resolution of the LULC classes, training data acquired in a field study could allow a more detailed analysis of the Landsat data. Additionally, further ground data, especially from the years 2000 to 2005, could allow a more detailed analysis of the drivers of land change and verify the impact of the Fast Track Land Reform.

\section{Acknowledgments}

This research was supported by the German Federal Ministry of Education and Research (grant number FKZ 01UC1201). The data ASTER GDEM is a product of METI and NASA, the precipitation data of Francistown were downloaded from www.ncdc.noaa.gov/cdo-web/datasets/GHCNDMS/ stations/GHCND:BC008948490/detail. The Landsat surface reflectance data were downloaded from earthexplorer.usgs.gov and are courtesy of the U.S. Geological Survey. We thank the anonymous reviewers for their constructive comments that helped to improve the manuscript.

\section{References}

Alexander, J., 2006. The Unsettled Land: State-making and the Politics of Land in Zimbabwe 1893-2003. James Currey. 
Anderson, I. P., Brinn, P. J., Moyo, M., Nyamwanza, B., 1993. Physical

Chen, X., Chen, J., Shi, Y., Yamaguchi, Y., 2012. An automated approach for updating land cover maps based on integrated change detection and classification methods. ISPRS Journal of Photogrammetry and Remote Sensing 71, 86 - 95 . 
Coppin, P., Jonckheere, I., K., N., Muys, B., 2004. Digital change detection methods in ecosystem monitoring: a review. International Journal of Remote Sensing 25 (10), 1565-1596.

DeFries, R., Hansen, A., Turner, B., Reid, R., Liu, J., 2007. Land use change around protected areas: management to balance human needs and ecological function. Ecological Applications 17 (4), 1031-1038.

Fernández-Delgado, M., Cernadas, E., Barro, S., Amorim, D., 2014. Do we need hundreds of classifiers to solve real world classification problems? Journal of Machine Learning Research 15, 3133-3181.

Fowler, P., 2002. World Heritage Cultural Landscapes 1992-2002. UNESCO World Heritage Centre.

Gaston, K. J., Jackson, S. F., Cantú-Salazar, L., Cruz-Piñón, G., 2008. The ecological performance of protected areas. Annual Review of Ecology, Evolution, and Systematics 39 (1), 93-113.

Gaveau, D. L. A., Curran, L. M., Paoli, G. D., Carlson, K. M., Wells, P., Besse-Rimba, A., Ratnasari, D., Leader-Williams, N., 2012. Examining protected area effectiveness in Sumatra: importance of regulations governing unprotected lands. Conservation Letters 5 (2), 142-148.

Gong, P., Wang, J., Yu, L., Zhao, Y., Zhao, Y., Liang, L., Niu, Z., Huang, X., Fu, H., Liu, S., et al., 2013. Finer resolution observation and monitoring 575 of global land cover: First mapping results with Landsat TM and ETM+ data. International Journal of Remote Sensing 34 (7), 2607-2654. 
Goslee, S. C., et al., 2011. Analyzing remote sensing data in R: the landsat package. Journal of Statistical Software 43 (4), 1-25.

Hastie, T., Tibshirani, R., Friedman, J., 2009. The Elements of Statistical Learning, 2nd Edition. Springer-Verlag New York.

Hentze, K., Thonfeld, F., Menz, G., 06 2016. Evaluating crop area mapping from MODIS time-series as an assessment tool for Zimbabwe's "Fast Track Land Reform Programme". PLoS ONE 11 (6), 1-22.

Hijmans, R. J., 2016. raster: Geographic Data Analysis and Modeling. R package version 2.5-8.

URL https://CRAN.R-project.org/package=raster

Jones, D. A., Hansen, A. J., Bly, K., Doherty, K., Verschuyl, J. P., Paugh, J. I., Carle, R., Story, S. J., 2009. Monitoring land use and cover around parks: A conceptual approach. Remote Sensing of Environment 113 (7), 1346 - 1356, Monitoring Protected Areas.

Juffe-Bignoli, D., Burgess, N., Bingham, H., Belle, E., de Lima, M., Deguignet, M., Bertzky, B., Milam, A., Martinez-Lopez, J., Lewis, E., Eassom, A., Wicander, S., Geldmann, J., van Soesbergen, A., Arnell, A., O’Connor, B., Park, S., Shi, Y., Danks, F., MacSharry, B., Kingston, N., 2014. Protected Planet Report 2014. UNEP-WCMC: Cambridge, UK.

Kaufman, L., Rousseeuw, P. J., 1990. Finding Groups in Data: An Introduction to Cluster Analysis. John Wiley \& Sons, Inc., Ch. Clustering large applications (Program CLARA), pp. 126-163. 
Knorn, J., Rabe, A., Radeloff, V. C., Kuemmerle, T., Kozak, J., Hostert, P., 2009. Land cover mapping of large areas using chain classification of neighboring landsat satellite images. Remote Sensing of Environment 113 (5), 957-964.

Kuhn, M., 2016. Classification and Regression Training.

Kuhn, M., Johnson, K., 2013. Applied Predictive Modeling, 1st Edition. 605 Springer-Verlag New York.

Liaw, A., Wiener, M., 2002. Classification and Regression by randomForest. R News 2 (3), 18-22.

Lu, D., Mausel, P., Brondízio, E., Moran, E., 2004. Change detection techniques. International Journal of Remote Sensing 25 (12), 2365-2401.

Pontius, R. G., Millones, M., 2011 2011. Death to Kappa: birth of quantity disagreement and allocation disagreement for accuracy assessment. International Journal of Remote Sensing 32, 4407 - 4429.

R Core Team, 2016. R: A Language and Environment for Statistical Computing. R Foundation for Statistical Computing, Vienna, Austria. URL https://www.R-project.org/

Ranger, T., JAN 1989. Whose heritage? The case of the Matobo National Park. Journal of Southern African Studies 15 (2), 217-249.

Schneider, A., Friedl, M. A., Potere, D., 2009. A new map of global urban extent from MODIS satellite data. Environmental Research Letters 4 (4), 044003. 
Sieber, A., Kuemmerle, T., Prishchepov, A. V., Wendland, K. J., Baumann, M., Radeloff, V. C., Baskin, L. M., Hostert, P., 2013. Landsat-based mapping of post-Soviet land-use change to assess the effectiveness of the Oksky and Mordovsky protected areas in European Russia. Remote Sensing of Environment 133, $38-51$.

Sokolova, M., Lapalme, G., 2009. A systematic analysis of performance measures for classification tasks. Information Processing \& Management 45 (4), $427-437$.

Strobl, C., Malley, J., Tutz, G., 2009. An introduction to recursive partitioning: rationale, application, and characteristics of classification and regression trees, bagging, and random forests. Psycological Methods 14 (4), $323-348$.

Sudhir Wanmali, Y. I., 1997. Rural infrastructure and agricultural development in Southern Africa: a centre-periphery perspective. The Geographical Journal 163 (3), 259-269.

Tewkesbury, A. P., Comber, A. J., Tate, N. J., Lamb, A., Fisher, P. F., 2015. A critical synthesis of remotely sensed optical image change detection techniques. Remote Sensing of Environment 160, 1 - 14 .

Wasiolka, B., Blaum, N., 2011. Comparing biodiversity between protected 640 savanna and adjacent non-protected farmland in the southern Kalahari. Journal of Arid Environments 75 (9), 836 - 841. 


\section{List of figure captions}

Figure 1 Partitioning of the study area with a Landsat image in the background, R: area outside the World Heritage Site, WH: World Heritage Site

645 without national park, NP: Matobo National Park, NP-margin: the outer $500 \mathrm{~m}$ of the national park. The extension of the common lands east of $29^{\circ} 03^{\prime} 23^{\prime \prime}$ is unknown.

Figure 2 Distribution of $\rho$ (gray line) and fit consisting of two Gaussians (black line). $\rho$ is the length of the change vector, which is calculated separately

650 for each pixel from the difference between the surface reflectance in each band of two Landsat images. It describes the degree of change in a pixel.

Figure 3 Photographs illustrating the four land use and land cover classes: shrub land (top left), forest (top right), patchy vegetation (bottom left) and 655 agricultural area (bottom right). (CKupakwashe Mtata)

Figure 4 Spatial distribution of four LuLC classes in the study area. The corresponding LULC classes are: 1: shrub land, 2: forest, 3: patchy vegetation, and 4: agricultural area.

Figure 4a 1989

Fingure 4b 1998

Figure 4c 2014

Figure 5 Fractions of the four LULC classes in the years 1989, 1998 and 2014 for the regions: Area outside the World Heritage Site (R), World Heritage 
Site without national park (WH), national park (NP). By adding the pixels with missing values (see south east of the image of 1989, Figure 4a) every bar sums up to 1 . The exact numbers can be found in Section 2.2 in the online Supplementary Material.

Figure 6 Fractions of LULC classes for a $500 \mathrm{~m}$ margin of NP and the core area. The exact numbers can be found in Section 2.2 in the online Supplementary Material.

Figure 7 Fractions of the four LULC classes in the years 1989, 1998 and 2014 for the regions: Area outside the World Heritage Site (R), and World Heritage Site without national park (WH), both distinguished for common land and not-common land. Region $\mathrm{R}$ was reduced to the part of $\mathrm{R}$ west of $29^{\circ} 03^{\prime 2} 23^{\prime \prime}$ due to missing information on the extension of common land in the East. The exact numbers can be found in Section 2.2 in the online Supplementary Material. 